\title{
Simulation of oil spill behaviour and response operations in PISCES
}

\author{
L. Delgado, E. Kumzerova \& M. Martynov \\ Transas Technologies, Russia
}

\begin{abstract}
The aim of this paper is to present a novel method of numerical simulation of oil spill behaviour in the conditions of human response.

The main point of the method is to extend the Lagrangian approach by introducing interactions between oil particles. This innovation allows some essential disadvantages of the traditional Lagrangian method to be compensated, in particular the impossibility of describing oil interaction with different kinds of natural and artificial barriers like coastlines and booms. The tuning of the interparticle interaction was made via multi-stage parametric optimization with the aid of the alternating-variable descent technique, and includes the verification with known semi-empirical solutions as well as with different logical tests.

The model takes into account the main physicochemical processes occurring in the oil slick, which include evaporation, dispersion, emulsification and viscosity variation. Simulation is carried out with regard to the following environment factors: coastline, field of currents, weather, sea state, ice conditions and environmentally sensitive areas. In addition, models of response resource application including booming and recovery have been developed.

The presented technique is used as model part of the PISCES (Potential Incident Simulation Control and Evaluation System) oil spill simulator produced by TRANSAS and designed for planning spill recovery after accidental marine oil spills.
\end{abstract}

Keywords: oil spill simulation, oil spreading model, response strategies.

\section{Introduction}

Numerical simulation of oil pollution fate is an important tool for the planning of spill recovery strategies. At the same time forecasting in actual situations taking 
into account possible human response is a challenging task. The following can be listed among the main difficulties:

- description of areas with complex geometry (of both, oil spill shape and actual coastline);

- wide range of initial spill parameters (from tons to thousands of tons) are to be simulated;

- necessity for detailed presentation on different scales: from tens of meters in the case of interaction with response equipment, up to tens of kilometres in the description of an accident region as a whole;

- dynamically changing environment (unsteady currents and weather conditions, response equipment transport);

- limited computational time for forecasting, and others.

An attempt to combine all the above requirements has been made in the presented oil model which is part of the PISCES simulator developed by TRANSAS.

This paper provides the up-to-date description of the PISCES oil model and has the objective to demonstrate that the proposed model does not only allow simulation of the oil spill behaviour with reasonable accuracy, but also enables development of recovery strategies within a wide range of oil spill initial parameters and environmental conditions.

\section{PISCES system}

The PISCES is an incident response simulator designed for preparing and conducting command centre exercises and area drills. The application is developed to support exercises focusing on oil spill response. Therefore, the oil spill mathematical model should take into account human response activities in addition to such environmental factors as coastline, field of currents, weather, sea state, ice conditions and environmentally sensitive areas.

The coastline is presented in the program as a set of closed polygons obtained automatically from vector-based nautical charts. Owing to a large number of these navigational charts, the PISCES enables description of the modelled area in practically any point of the globe to be generated promptly and with a required accuracy.

The field of currents is determined from a set of "base vector" stations with the given time-dependent velocity variations. The velocity of the surface current at a certain point is determined by using the linear interpolation of base vector values on the triangular mesh based on Delaunay principle.

Environmentally sensitive areas are presented as polygons with a certain number of dweller groups. The number of living organisms and their lethal concentration of toxic substances are specified for each group.

Two kinds of ice are considered in PISCES: pack and fast ice. Ice is presented as a set of polygons. For the fast ice the shape and location of ice regions, ice thickness and age are to be specified. The pack ice has the ice concentration in addition to the same parameters as for the fast ice. Ice concentration is 
understood as the ratio of area covered by ice to the whole area of pack ice region.

Simulation of human activities includes modelling of various types of response resources including booms and skimmers interacting with oil spill.

\section{Physical model description}

A great number of oil spill models have been developed in the last decades (see the recent reviews [1-3]).

At the present time, the most popular models of oil spreading are semiempirical models by Fay [4], Mackay et al. [5] and Lehr et al. [6]. They consider oil spreading as one-dimensional process. The spreading of oil according to Fay is usually treated as a process consisting of three stages. The gravity-inertial phase usually lasts a few minutes, the gravity-viscous phase defines the slick spreading in several (up to 20) hours after the spill and then the tension-viscous phase takes place when the slick can be broken in parts, dispersed etc.

Two approaches to computing oil spill trajectories are normally applied. These models are based on the Lagrangian or on the Eulerian approach.

The equations in Eulerian approach are based either on the mass and momentum conservation equations applied to the oil slick [7,8], or on a convection-diffusion equation $[9,10]$, in which the diffusive part of the equation represents the spreading of oil by itself and the convective terms represent advection of oil by currents and winds.

The Lagrangian models [11,12] consist in representing the oil slick by an ensemble of a large number of particles advected by the surface motion resulting from the combined effect of winds, currents and diffusion.

The Lagrangian models have proved to be more suitable for simulation of actual situations, being simpler, more efficient, and less costly computationally than the Eulerian models.

At the same time this method has some limitations in simulating oil interaction with various barriers (e.g., coastline, boom, etc.), which is especially important in the case of response operation modelling.

An extension of the above method is proposed below. It is based on the idea of implementing interactions between oil particles.

\subsection{Oil transport}

The model represents an oil spill as a number of particles assumed to have an identical set of properties. The number of particles depends on the desired level of detail and can be changed during the simulation. In this case re-calculations are made, based on the interpolation or extrapolation of geometrically closest neighbours and on the combining of their attributes such as mass, time since release and location.

Based on four mechanisms of oil spill movement, namely the determinate (currents, wind and spreading mechanisms) and the stochastic process, and also 
assuming the process to be two-dimensional, the transport equation for a single particle can be written in the following form:

$$
\frac{\partial \vec{X}_{i}}{\partial t}=\vec{V}_{d r i f t}\left(x_{i}, y_{i}, t\right)+\vec{V}_{\text {diff }}\left(x_{i}, y_{i}, t\right)+\vec{V}_{\text {spread }}\left(x_{i}, y_{i}, t\right),
$$

where $\vec{X}_{i}=\left(x_{i}, y_{i}\right)$ is location of $i^{\text {th }}$ particle, $t$ is time (s), $\vec{V}_{d r i f t}$ is drift velocity depending on the currents and wind, $\vec{V}_{\text {spread }}$ is spreading component and $\vec{V}_{\text {diff }}$ is diffusive component accounting for the stochastic process.

The drift velocity affecting mainly the oil transport can be calculated as:

$$
\vec{V}_{d r i f t}(x, y)=a_{w} \vec{V}_{w}(x, y)+a_{c} \vec{V}_{c}(x, y),
$$

where $\vec{V}_{w}$ is wind velocity, $\vec{V}_{c}$ is depth averaged pre-calculated velocity of current, $a_{w}=0.03$ is wind factor, $a_{c}=1.0$ is current factor.

The diffusive velocity component is simulated by using random walk approach [13]:

$$
\vec{V}_{\text {diff }}(x, y)=\vec{V}_{\text {drift }} R_{n} \exp \left(i \alpha_{n}\right)
$$

where $R_{n}$ is normally distributed random number within [-1,1], angle $\alpha_{n}$ is uniformly distributed random angle within the range of $[0, \pi)$.

Spreading component $\vec{V}_{\text {spread }}$ is computed as described in the next section.

\subsection{Model for spreading component}

In this model, a single particle has an additional property as compared to ordinary Lagrangian methods, namely a capability to interact with other particles and barriers. The process of particle interaction is assumed to be responsible for the spreading component.

We also assume that the spreading process is affected by:

- $\quad$ area of a single particle $S$ changing due to viscous and gravity forces;

- $\quad$ friction force between the particle and water surface.

It is worth noting that the aforementioned physical laws should describe qualitative trends of particle behaviour only. Quantitative adjustment is achieved by introducing model coefficients and parametric functions. These functions are found later by using the model tuning algorithm.

We assume that particles do not interact with each other unless when they are closer than $r_{0}$, where $r_{0}$ is the characteristic particle size proportional to $\sqrt{S}$. The force of oil particle interaction is directed along the axis connecting their centres and is equal to

$$
\vec{F}_{\text {int }}=g_{1}\left(K_{1}, K_{2}, r_{0}\right)\left(\frac{r_{0}}{|\vec{r}|}\right)^{q} \frac{\vec{r}}{|\vec{r}|} .
$$


That is, this force depends on the distance between particle centres $|\vec{r}|$ and presents an analogy of particle interaction with Sutherland potential. Here and later $K_{j}(j=1 . . n)$ and $g_{k}(k=1,2,3)$ are respectively coefficients and functions to be found.

When moving on the water surface, an oil particle is affected by the friction force which is taken into account as:

$$
\vec{F}_{C_{D}}=-g_{2}\left(K_{3}, K_{4}, m_{p}\right) m_{p} \vec{V}_{\text {spread }},
$$

where $m_{p}$ is the particle mass.

The second Newton law is used for determining the spreading velocity:

$$
m_{p} \frac{d}{d t} \vec{V}_{\text {spread }}=\vec{F}_{C_{D}}+\sum_{i=1}^{N_{\text {int }}} \vec{F}_{\text {int }}^{i},
$$

where $N_{\text {int }}$ is the number of particles interacting with the one considered at a certain moment of time.

The equation for evolution of particle area $S$ is written in the following way:

$$
m_{p} \frac{d^{2} S}{d t^{2}}=K_{5} \frac{m_{p}}{S}-g_{3}\left(K_{6}, K_{7}, m_{p}, S\right) \frac{d S}{d t} .
$$

\subsection{Description of weathering models}

The oil product is represented in the same way as in ADIOS oil library and characterized by a common product name, type (e.g., crude or refined), specific gravity, surface tension, viscosity, distillation curve, emulsification constant, pour and flash points.

A brief description of the basic weathering processes included in the model is provided below.

\subsubsection{Evaporation}

Evaporation process is simulated by using pseudo-component approach. The evaporation rate for a single component is written in the following form [14]:

$$
\frac{d Y}{d t}=A \frac{K_{m} v \chi P_{v}}{R_{g} T_{w}}
$$

where $Y$ is fraction volume $\left(\mathrm{m}^{3}\right), A$ is slick area $\left(\mathrm{m}^{2}\right), K_{m}$ is mass transfer coefficient for the evaporation $(\mathrm{m} / \mathrm{s}), v$ is molar fraction volume $\left(\mathrm{m}^{3} / \mathrm{mol}\right), \chi$ is molar concentration, $P_{v}$ is vapour pressure $(\mathrm{Pa}), R_{g}$ is gas constant ( $\left.R_{g}=8.31 \mathrm{KJ} / \mathrm{mol} \mathrm{K}\right), T_{w}$ is water temperature $(\mathrm{K})$. Correlations for molar volume and molar concentration are taken from ADIOS model. Mass transfer coefficient is taken to be a function of wind speed $V_{w}$ [14]:

$$
K_{m}=0.002 V_{w}^{0.78} \text {. }
$$




\subsubsection{Emulsification}

This process consists in the penetration of water in the oil. The emulsification does not normally start until a certain amount of oil has evaporated. In the model, the emulsification process starts as soon as the fraction of evaporated oil reaches the emulsification constant $K_{e m}$, which is determined from the experimental data.

Mackay's approach is used for describing emulsification rate [5]:

$$
\frac{d F_{e m}}{d t}=K_{e m} \cdot\left(1+V_{w}\right)^{2} \cdot\left(1-\frac{F_{e m}}{F_{e m}^{f}}\right),
$$

where $F_{e m}$ is water volume fraction, $K_{e m}$ is a constant equal to $2 \cdot 10^{-6}\left(\mathrm{~s} \cdot \mathrm{m}^{-2}\right)$. $F_{e m}^{f}$ is the parameter corresponding to the final water volume fraction and depending on the oil type. $F_{e m}^{f}$ is assumed to be equal to 0.7 for crude oils and 0.25 for refined products.

\subsubsection{Dispersion}

The following expression for natural oil dispersion rate is implemented in the model [5]:

$$
\frac{d m_{d}}{d t}=K_{d} m_{\text {oil }} \frac{\left(1+V_{w}\right)^{2}}{1+5000 \eta^{1 / 2} h \sigma},
$$

where $m_{d}$ is dispersed oil mass $(\mathrm{kg}), m_{\text {oil }}$ is remaining oil slick mass $(\mathrm{kg}), \sigma$ is oil-water surface tension (dyne/cm), $\eta$ is dynamic viscosity $(\mathrm{cP})$, $K_{d}=3.05 \cdot 10^{-5}$.

\subsubsection{Viscosity variation}

The increase of oil viscosity is the result of emulsion formation, evaporation of light fractions and temperature drop. Viscosity variation due to emulsion formation is defined by Mooney equation [15], and the evaporation effect can be taken into account following [5]. The final correlation for viscosity variation is expressed as:

$$
\eta=\eta_{0} \exp \left[\frac{C_{V} F_{e m}}{1-C_{M} F_{e m}}+C_{E} F_{e v}+C_{T}\left(\frac{1}{T}-\frac{1}{T_{0}}\right)\right] .
$$

where $T_{0}$ is temperature corresponding to the initial oil viscosity $\eta_{0}, T$ is current oil temperature (K), $C_{V}, C_{M}, C_{T}$ are empirical constants, $C_{V}=2.5$ [5], $C_{M}=0.65$ [15] and $C_{T}=5.0$ [5]. Constant $C_{E}$ varies from 1 for crude oils to 10 for refined oils.

\subsection{Oil in ice conditions}

This part of the model has been designed primarily for illustrating qualitatively the main behaviour features of an oil spill in ice conditions. 
The presence of ice has a considerable effect on oil transport and spreading as well as on weathering processes. Moreover, it necessitates simulation of oil encapsulation into ice.

The basic features of additions to the model are described below.

\subsubsection{Solidification}

In the cold conditions under consideration it is necessary to take into account the effect of air temperature. We assume that oil spreading and weathering processes stop when the air temperature reaches the oil pour point value.

\subsubsection{Transport and spreading}

Within the pack ice area, oil moves with currents and wind at a velocity smaller than in the open water. Spreading is also decreased and can be described by modified Fay approach [16], and is expressed as:

$$
A_{\text {ice }}=\left[\frac{\eta_{o}}{\eta_{w}}\right]^{-0.15} \cdot\left(1-f_{i c e}\right) \cdot A_{F a y},
$$

where $\eta_{w}$ is the dynamic viscosity for water, $f_{i c e}$ is the ice concentration, $A_{i c e}$ is the oil spill area in ice conditions, $A_{F a y}$ is the area according to aforementioned Fay or Mackay solutions.

Under the fast ice, oil moves and also spreads at a velocity lower than in the open water. The decrease in the movement and spreading speed depends on the ice thickness and oil thickness.

The spreading stops when oil thickness reaches the critical value [16]:

$$
h_{\min }^{\text {under_ice }}=\sqrt{\frac{2 \sigma}{\left(\rho_{w}-\rho_{o}\right) g}},
$$

where $\rho_{w}, \rho_{o}$ are the water and oil densities $\left(\mathrm{kg} / \mathrm{m}^{3}\right), g$ is gravitation constant $\left(9.81 \mathrm{~m} / \mathrm{s}^{2}\right)$.

\subsubsection{Weathering processes}

Within the fast ice area, weathering processes are assumed not to take place as we consider oil moving under ice.

In the area of drift ice, oil evaporation rate changes $\left(1-f_{\text {ice }}\right)$ times due to the reduction of air contact area, while emulsification and dispersion rates remain the same.

\subsubsection{Oil encapsulation into ice}

When oil interacts with any type of ice, the floating oil mass decreases due to the absorption by ice. The following model for taking into account this effect has been implemented:

$$
\frac{d h_{\text {freez }}}{d t}=K_{\text {freez }}\left(1-\frac{h_{\text {freez }}}{h_{\text {ice }}}\right),
$$


where $h_{\text {freez }}$ and $h_{\text {ice }}$ define oil encapsulation thickness and ice thickness respectively, coefficient $K_{\text {freez }}$ expresses the velocity of movement through ice and is within range of $0.1-50 \mathrm{~mm} /$ hour [16].

\subsection{Model tuning and verification}

According to the model description we have $K_{1} . . K_{n}$ coefficients and functions $g_{1} . . g_{3}$ to be found to approximate the oil spill behaviour with the highest possible accuracy.

To find these coefficients and functions the following idea is applied:

- $\quad$ to fix a number of test examples and formulate criteria of algorithm;

- $\quad$ to introduce a special function of the algorithm accuracy as a whole and to apply multi-stage parametric optimization depending on the introduced coefficients.

Four numbers of test cases were identified as the main ones:

- $\quad$ Test on point source spreading velocity in case of absence of wind. This implies that a point source should spread according to known analytical solution (e.g. [4,5]).

- $\quad$ Test on identity of short leak and point sources. It is expected that two sources with the same mass, one is the point source, another one is the leak source should approach each other after a certain time moment.

- Scaling test. This implies that the adjusted coefficient should affect linearly the spill radius.

Test on downwind elongation. Under wind conditions the spill considered is assumed to elongate according to known analytical solution (e.g. [6]).

Accuracy criteria of each test have been defined as an average sum of timeaveraged relative discrepancy from every sample.

The total algorithm correction function has been defined as a linear combination of correction functions of each test.

The parametric optimization has been made with the aid of alternatingvariable descent technique, and provided below are some illustrations of this process results.

Fig.1 illustrates the behaviour of point (lower) and short leak (upper) sources with the following parameters: oil source mass is 100 tons, mass rate of leak source is 1200 tons per hour which corresponds to 5 minutes of leakage. Acceptable agreement can be seen for both slick area and slick shape as early as 2 hours after the spillage. The relative area discrepancy at this time is only about 8 percent.

Fig.2 demonstrates reasonable agreement between PISCES simulations and analytical solution of Mackay [5] for a large amount of initial parameters of an oil spill. It can bee seen that in the case of spills of less than 1000 tons, instant deviation from the aforementioned approximation is less than 9 percent. 
00:05

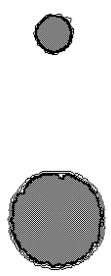

$00: 30$
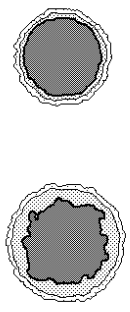

1:00
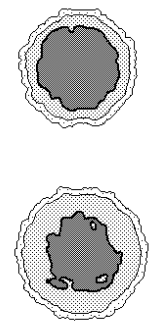

2:00
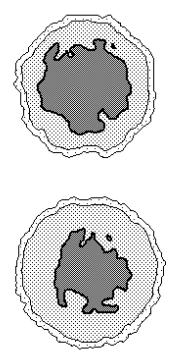

Figure 1: Evolution of point and leak sources in the course of 2 hours after the spillage.

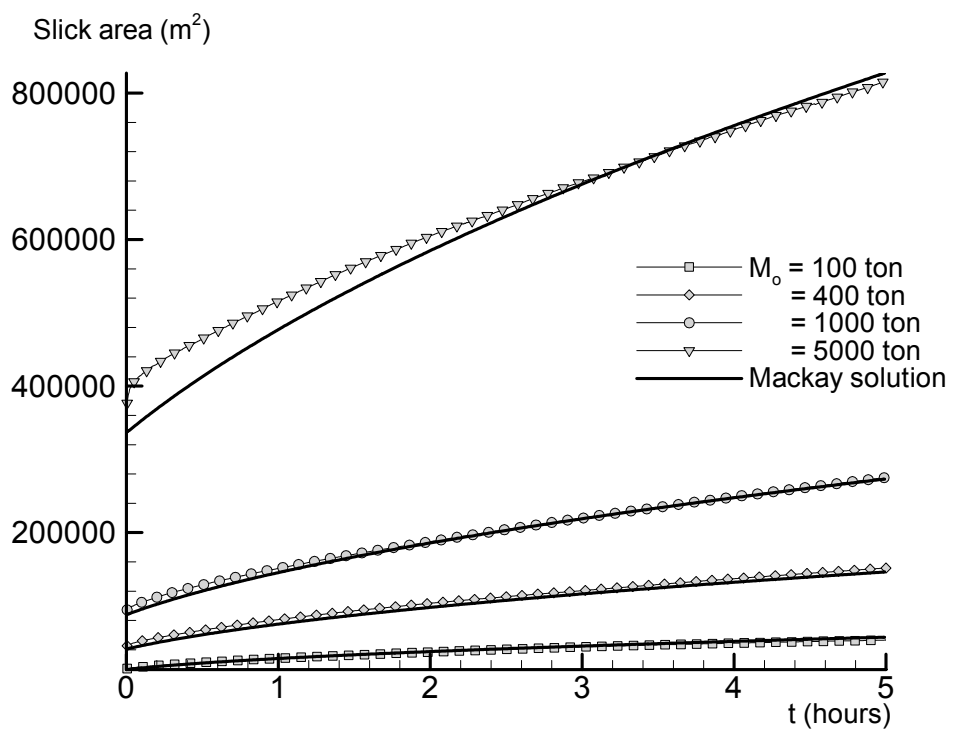

Figure 2: Time variation of slick area for different initial oil masses according to the model simulation (dots) and Fay solution (line). Oil density is $851 \mathrm{~kg} / \mathrm{m}^{3}$.

The above illustrations allow making a conclusion about the adequacy of the spreading model. The adequacy of the whole model of oil behaviour can be proved by detailed testing and verification of each model component, by comparing it with experimental data for weathering models (see [17]) as well as by comparing with other available model data (ADIOS and OSIS oil models). 


\section{Biological model}

The biological model estimates toxic response of marine biota to the spilled oil. The model considers several groups of organisms, amphibians, annelids, fishes, mammals, molluscs, phytoplankton, zooplankton and birds among them. Each group is characterized by toxic concentration $\mathrm{LC}_{50}$, lethal to $50 \%$ of exposed organisms during time of exposure $\mathrm{T}_{\mathrm{LC} 50}$.

The mortality of a group is assumed to be a function of oil concentration $c$ and time of exposure $t$ only. In a simplified case, when oil concentration does not depend on time, the number of dead individuals $N_{\text {mort }}$ can be expressed as:

$$
N_{\text {mort }}(c, t) \sim-\exp \left(-\frac{t}{t_{0}}\right) \cdot \exp \left(-\frac{c}{c_{0}}\right)
$$

where $t_{0}, c_{0}$ correspond to the characteristic time and concentration of the considered process.

The function $N_{\text {mort }}$ should meet the following requirements:

$$
\bullet
$$$$
\text { initial condition: } N_{\text {mort }}(0,0)=N_{0} \text {, }
$$$$
\text { definition of lethal concentration } L C_{50} \text { : }
$$$$
N_{\text {mort }}\left(L C_{50}, T_{L C_{50}}\right)=\frac{1}{2} N_{0} .
$$

According to the above conditions the final correlation for function (16) used in calculations was found to be:

$$
N_{\text {mort }}(c, t)=N_{0}\left[1-2 \exp \left(-b\left(\frac{t}{T_{L C_{50}}}+\frac{c}{L C_{50}}\right)\right)\right],
$$

where $b$ is a constant $(b=\ln 2 \approx 0.693)$.

\section{Response operations}

\subsection{Booms}

Booms are simulated as flexible permeable barriers with an ability to move. The permeability or efficiency of booms is calculated with regard to the boom type, wave height and relative water velocity.

Boom shape is assumed to depend on currents and wind. Drag force affecting the boom on a unit of length is the sum of current and wind drag forces:

$$
\vec{F}=\vec{F}_{c}+\vec{F}_{w},
$$

which are represented respectively by the expressions:

$$
\vec{F}_{c}=C_{n} \rho_{w} H_{w}\left|\vec{V}_{d r i f t}\right| \vec{V}_{d r i f t}, \vec{F}_{w}=C_{n} \rho_{a} H_{a}\left|\vec{V}_{w}\right| \vec{V}_{w},
$$


where $C_{n}$ is drag coefficient, $\rho_{a}$ is air density $\left(\mathrm{kg} / \mathrm{m}^{3}\right), \quad H_{a}$ is boom height above the sea level (m), $H_{w}$ is boom depth below the sea level (m).

After the main force vector has been defined, the problem in the boom reference system is reduced to solving a catenary problem:

$$
z(u)=a \cosh \left(\frac{u-b}{a}\right)+c,
$$

where axis $z$ coincides with the main force vector direction. Parameters $a, b$ and $c$ are determined numerically from boundary conditions.

\subsection{Skimmers}

Skimmer is considered to be a negative source in the model and has the following parameters: $E_{s k}$ is skimmer efficiency which determines the skimmer productivity reduction; $\operatorname{Pr}_{s k}$ is rated skimmer productivity $(\mathrm{kg} / \mathrm{s}) ; E_{S S}$ is water volume fraction in oil-water mixture additionally removed by the skimmer and depending on the wave height; $E_{\eta}$ is efficiency coefficient depending on the oil viscosity.

The rate of oil mass recovery is calculated as:

$$
R_{r e c}=E_{s k} E_{\eta}\left(1-F_{e m}\right)\left(1-E_{S S}\right) \operatorname{Pr}_{s k},
$$

where $F_{e m}$ is average volume fraction in the vicinity of the skimmer. Maximum oil mass accumulated by the skimmer is limited by the skimmer capacity $C\left(\mathrm{~m}^{3}\right)$.

\section{Examples}

Some illustrations of possible response strategies simulated in PISCES are presented below.

Fig. 3 demonstrates containment of oil spill propagation with the aid of booms. It can be seen that oil patches partially go through the booms due to their limited retention capability. Skimmer operation in the second booming order results in the local thinning of the oil slick. Fig. 4 presents a hypothetical oil spill interacting with several stationary booms and complicated coastline in the port of Helsinki area.

\section{Conclusions}

The description of the PISCES oil model is presented and includes the following features:

- $\quad$ model description of oil processes on the sea surface;

- description of biological impact model;

- description of environment presentation including coastline, field of currents, weather, ice conditions and environmentally sensitive areas;

- $\quad$ simulation of cleanup operations such as booming and skimming. 
290 Environmental Problems in Coastal Regions VI

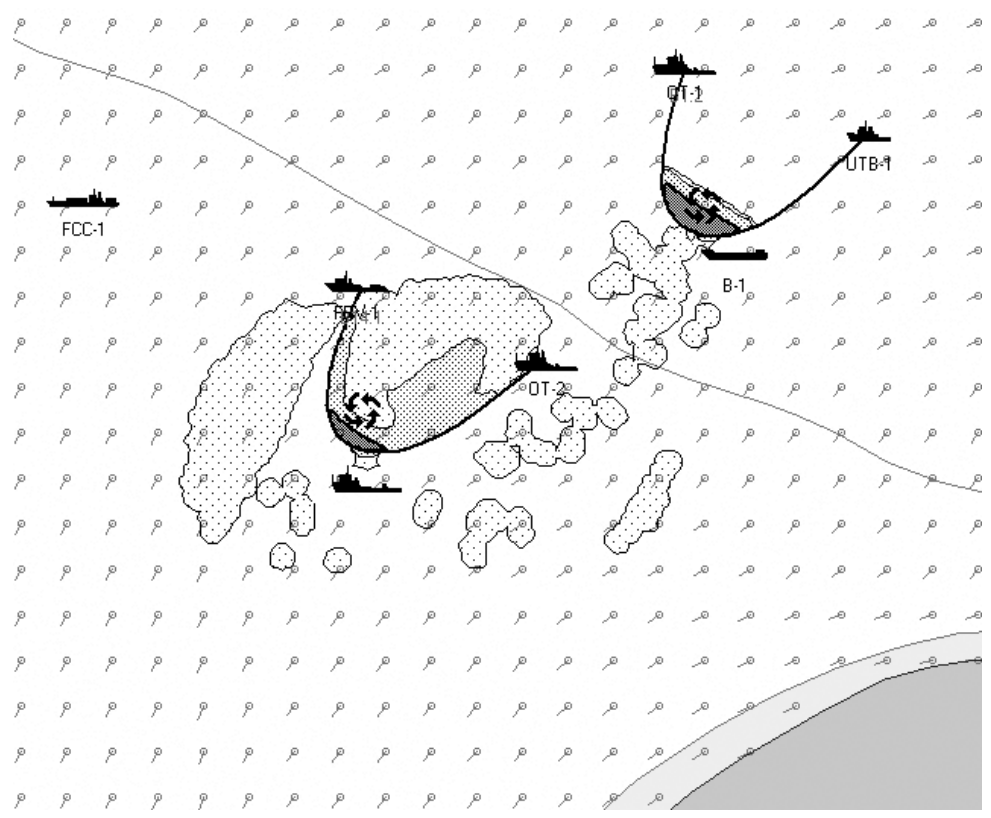

Figure 3: Illustration of booming and on-water recovery operation.

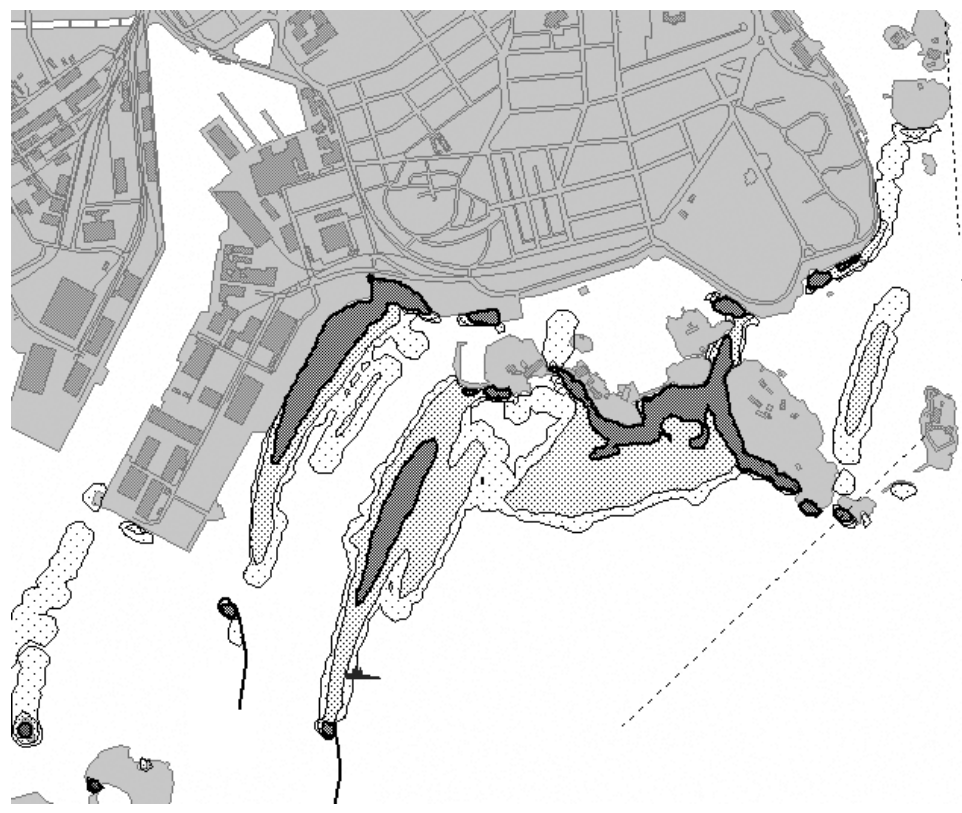

Figure 4: Simulation of an oil spill in port of Helsinki, Finland. 
It has been demonstrated that owing to the fact that particle interactions were taken into account in the Lagrangian part of the model, the developed technique has some essential advantages. In particular, the reasonable accuracy shown in several verification tests combines with the possibility of simulation complicated flow structures and interaction with coastline and booms.

It is also worth noting that computational time has not increased significantly as compared to the ordinary particle-tracking method. This method thus offers a promising approach to carrying out table-top oil spill response exercises whose objective is to demonstrate several examples of the oil spill fate under the circumstances of different response strategies.

\section{References}

[1] Reed, M., Johansen, Ø., Brandvik, P.J., Dailing, P., Lewis, A., Fiocco, R., Mackay, D., Prentki, R., Oil spill modelling towards the close of the 20th century: overview of the state-of-the-art. Spill Science Technology Bulletin, 5, pp. 3-16, 1999.

[2] Lehr, W.J., Review of modelling procedures for oil spill weathering behaviour. Oil Spill modelling and processes, ed. Brebbia, C.A. WIT Press, pp. 51-90, 2001.

[3] Cekirge, H.M., Koch, M., Long, C., Giammona, C. P., Binkley, K., Engelhardt, R., Jamail, R., State-of-the-art technologies in oil spill modelling. Proceedings of International Oil Spill Conference 2003, Vancouver, Canada, 2082, 2003.

[4] Fay, J.A., Physical processes in the spread of oil on a water surface. Proceedings On Prevention and Control of Oil Spill, American Petroleum Institute: Washington, DC, pp. 463-467, 1971.

[5] Mackay, D., Buistt, I.A., Marcarenhas, R., Paterson, S., Oil spill processes and models. Environment Canada Manuscript Report No. EE-8, Ottawa, Ontario, 1980.

[6] Lehr, W.J., Fraga, R.J., Belen, M.S., Cikerge, H.M., A new technique to estimate initial spill size using a modified Fay-type spreading formula. Marine Pollution Bulletin, 15 (9), pp. 326-329, 1984.

[7] Hess, K. W., Kerr, C. L., A Model to Forecast the Motion of Oil on the Sea. Proceedings of the Oil Spill Conference, pp. 653-663, 1979.

[8] Ovsienko, S., Zatsepa, S., Numerical simulation features of initial stage spreading of marine oil spill. Oceanographic aspects of chemical pollution control of seas and oceans (in Russian), Gidromet: Moscow, 1989.

[9] Venkatesh, The Oil Spill Behaviour Model of the Canadian Atmospheric Environment Service, part 1. Atmosphere.Ocean, 26, pp. 93-108, 1988.

[10] Tkalich, P., Xiaobo, C., Accurate simulation of oil slicks. Computer technology and oil spills, pp. 1133-1137, 2001.

[11] Shen, H. T., Yapa, P. D., Oil Slick Transport tin Rivers. Journal of Hydraulic Engineering, 114 (5), pp. 529-543, 1988. 
[12] Lardner, R.W., Lehr, W., Fraga, R., Sarhan, M., A model of residual currents and pollution transport in the Arabian Gulf. Applied mathematical modelling, 12, pp. 379-389, 1988.

[13] Csanady, G.T., Turbulent diffusion in the environment, D. Reidel Publishing Company, Dordrecht, Holland, 248p, 1973.

[14] Stiver, W., Mackay, D., Evaporation rate of spills of hydrocarbons and petroleum mixtures. Environmental Science and Technology, 18, pp. 834840, 1984.

[15] Mooney, M., The viscosity of a concentrated suspension of spherical particles. J. Colloidal Science, 10, pp. 162-170, 1951.

[16] Fingas, M.F., Hollebone, B.P., Review of behaviour of oil in freezing environments. Marine pollution bulletin, 47, pp. 333-340, 2003.

[17] Delgado, L., Kumzerova, E., Martynov, M., Mirnyj, K., Shepelev, P., Dynamic simulation of marine oil spills and response operations. Coastal Engineering VII. Modelling, Measurements, Engineering and Management of Seas and Coastal Regions, Ed: Brebbia C.A., M. de Conceicao Cunha, WIT Press, pp. 123-133, 2005. 